ROBERTO EDUARDo BitTAR ${ }^{1}$

Marcelo ZugaiB ${ }^{2}$

\title{
Tratamento do trabalho de parto prematuro
}

\author{
Management of preterm labor
}

Revisão

Palavras-chave

Tocólise

Tocolíticos/uso terapêutico Trabalho de parto prematuro/prevenção

\& controle

Trabalho de parto prematuro/

quimioterapia

Contração uterina/efeito de drogas

Corticosteróides/administração \& dosagem

Keywords

Tocolysis

Tocolytic agents/therapeutic use Obstetric labor, premature/prevention \&

control

Obstetric labor, premature/drug therapy

Uterine contraction/drug effects

Adrenal cortex hormones/administration \& dosage
Correspondência:

Roberto Eduardo Bitto Clínica Obstétrica do Instituto Central do Hospital das Clínicas Avenida Doutor Enéas de Carvalho Aguiar, 255,

$10^{\circ}$ andar - Cerqueira César CEP 05403-000 - São Paulo (SP), Brasi E-mail: roberto.bittar@hcnet.usp.br

Recebido

$14 / 7 / 09$

\section{Resumo}

O objetivo principal para o uso de uterolíticos no trabalho de parto prematuro é prolongar suficientemente a gestação para a administração materna de glicocorticoides e/ou realizar a transferência materna para um centro hospitalar terciário. As decisões sobre o uso e a escolha de uterolítico requerem o diagnóstico correto do trabalho de parto prematuro, o conhecimento da idade gestacional, das condições médicas materno-fetais, da eficácia, dos efeitos colaterais e do custo do medicamento. Todos os uterolíticos possuem efeitos colaterais e alguns deles são potencialmente letais. Os estudos sugerem que os agonistas de receptores beta-adrenérgicos, os bloqueadores de cálcio e os antagonistas de receptor de ocitocina são eficazes para prolongar a gestação por pelo menos 48 horas. Dos três agentes, o atosiban (antagonista de receptor de ocitocina) possui maior segurança, embora o cusło seja elevado. $\bigcirc$ sulfało de magnésio não é eficaz para prolongar a gestação e apresenta efeitos colaterais importantes. Os inibidores da ciclooxigenase também apresentam efeitos colaterais significativos. Até o momento, não há evidências suficientes para se recomendar o uso de doadores de óxido nítrico para inibir o trabalho de parto prematuro. Não existem fundamentos para o emprego de antibióticos para evitar a prematuridade diante do trabalho de parto prematuro.

\section{Abstract}

The main purpose of using uterulytic in preterm delivery is to prolong gestation in order to allow the administration of glucocorticoid to the mother and/or to accomplish the mother's transference to a tertiary hospital center. Decisions on uterolytic use and choice require correct diagnosis of preterm delivery, as well as the knowledge of gestational age, maternal-fetal medical condition, and medicine's efficacy, side-effects and cost. All the uterolytics have side-effects, and some of them are potentially lethal. Studies suggest that beta-adrenergic receptor agonists, calcium blockers and cytokine receptor antagonists are effective to prolong gestation for at least 48 hours. Among these three agents, atosiban (a cytokine receptor antagonist) is safer, though it presents a high cost. Magnesium sulfate is not efficient to prolong gestation and presents significant side-effects. Cyclooxygenase inhibitors also present significant side-effects. Up till now, there is not enough evidence to recommend the use of nitric oxid donors to inhibit preterm delivery. There is no basis for the use of antibiotics to avoid prematurity in face of preterm labor.
Departamento de Obstetrícia e Ginecologia da Faculdade de Medicina da Universidade de São Paulo - USP - São Paulo (SP) Brasil.

'Professor-associado da Disciplina de Obstetrícia do Departamento de Obstetrícia e Ginecologia da Faculdade de Medicina da Universidade de São Paulo - USP - São Paulo (SP), Brasil.

2 Professor Titular da Disciplina de Obstetrícia do Departamento de Obstetrícia e Ginecologia da Faculdade de Medicina da Universidade de São Paulo - USP - São Paulo (SP), Brasil. 


\section{Introdução}

O trabalho de parto prematuro (TPP) é responsável por $75 \%$ dos nascimentos antes da $37^{\mathrm{a}}$ semana de gestação. A sua prevenção durante o pré-natal é poucas vezes possível, pois, geralmente, apresenta etiologia multifatorial ou desconhecida. Dessa maneira, a inibição das contrações uterinas ou tocólise é o último recurso preventivo disponível ao obstetra para adiar o nascimento. Sabe-se que a tocólise comumente não evita o parto prematuro, pois as causas desencadeantes do TPP persistem e, na maioria das vezes, é possível apenas abolir as contrações uterinas por algum tempo. No entanto, quando se pode adiar o parto por pelo menos 48 horas, torna-se possível a realização da corticoterapia antenatal e, com isso, a redução do risco de complicações neonatais, tais como a síndrome do desconforto respiratório (SDR), a hemorragia cerebral, a enterocolite necrosante e morte neonatal. Além disso, tal medida permite uma eventual transferência da gestante para outro serviço hospitalar com melhores condições de atendimentos a prematuros.

\section{Diagnóstico do trabalho de parto prematuro}

A primeira dificuldade é o reconhecimento do início do trabalho de parto (TP). O útero apresenta atividade contrátil durante toda a gestação, com contrações de dois tipos: de baixa e de grande amplitude (Braxton Hicks). No último trimestre da gestação, as contrações de Braxton Hicks vão se tornando cada vez mais frequentes e podem ser confundidas com contrações de trabalho de parto. Tal fato é responsável pelas internações desnecessárias em cerca de metade das gestantes supostamente em TPP ${ }^{1}$.

As dificuldades existentes para o diagnóstico inicial do TPP fazem com que até o momento não exista protocolo, baseado em evidências, sobre quais alterações de contratilidade uterina e do colo uterino justificam a realização do tratamento. Dessa maneira, os critérios para o diagnóstico do TPP variam na literatura ${ }^{2}$. Serão apresentados, a seguir, os critérios mais aceitos para o diagnóstico de TPP e contrações uterinas regulares a cada cinco minutos. O American College of Obstetricians and Gynecologists enfatiza a importância da persistência das contrações, ou seja, pelo menos quatro contrações em 20 minutos ou oito contrações

Quadro 1 - Diferenças clínicas entre trabalho de parto e falso trabalho de parto.

\begin{tabular}{|ll|}
\hline Trabalho de parto & Falso traballho de parto \\
\hline Contraç̃ões em intervalos regulares & Falso trabalho de parto \\
Contraç̃oses com intensidade crescente & Intensidade variável \\
Dores predominantemente na região sacral & Dores abdominais \\
Aumento das contraç̃oes ao deambular & O deambular não exerce efeito \\
Esvaecimento e dilatação progressivos do colo & Esvaecimento e dilatações: se presentes, não \\
uterino & evoluem \\
\hline
\end{tabular}

em 60 minutos $^{2}$; dilatação cervical de pelo menos $1 \mathrm{~cm} \mathrm{e}$ presença de esvaecimento cervical.

No falso trabalho de parto (FTP), não ocorre mudança progressiva do colo, e as contrações cessam espontaneamente após um período de observação (Quadro 1). Para o diagnóstico diferencial em casos duvidosos, é importante que a gestante seja observada durante duas ou três horas antes de iniciar a tocólise. Outros recursos que também podem ser utilizados nos casos em que há dúvidas são: o teste para detecção da fibronectina fetal (fFN) e a ultrassonografia transvaginal para medida do comprimento do colo (CC) uterino. Quando o teste é positivo para fFN e a medida do CC uterino é menor que $15 \mathrm{~mm}$, é altamente provável que o parto ocorra. Por outro lado, o teste da fFN negativo e a medida do CC uterino igual ou maior que $15 \mathrm{~mm} \mathrm{FTP}^{3}$.

Infelizmente, a dificuldade de diagnóstico do verdadeiro TPP e os diferentes critérios utilizados para tanto tornam difícil a interpretação dos resultados de trabalhos científicos sobre os distintos tipos de uterolíticos.

\section{Avaliação inicial}

Antes de instituir a terapêutica inibitória, é preciso atentar para as indicações e contraindicações da tocólise (Quadros 2 e 3). A gestante deve ser hospitalizada e as providências e avaliações descritas a seguir devem ser realizadas ${ }^{4}$ :

Vitalidade fetal: desde que haja viabilidade fetal, ou seja, idade gestacional igual ou superior a 26 semanas, deve ser realizada a cardiotocografia fetal. Posteriormente, durante a tocólise, os batimentos cardíacos fetais também devem ser monitorados em intervalos regulares. Com tal medida, também são monitoradas as contrações uterinas.

Ultrassonografia deve ser feita para confirmar a apresentação fetal, analisar o volume de líquido amniótico, estimar o peso do feto, a idade gestacional e pesquisar possíveis malformações.

Exame bacterioscópico e cultura de conteúdo vaginal e anal para o Estreptococo do Grupo B e coleta do conteúdo cervical para pesquisa de Chlamydia trachomatis e Neisseria gonorrhoea devem ser realizados.

Acesso venoso e coleta de hemograma e de urina para exame de urina tipo I e cultura de urina também devem ser feitos.

\section{Tratamento do trabalho de parto prematuro}

\section{Limites inferiores e superiores da idade gestacional para a tocólise}

A idade gestacional mínima para se realizar a tocólise é motivo de controvérsia e não existem recomendações baseadas em evidências ${ }^{5}$. Alguns autores consideram 15 semanas o ideal, momento em que as perdas decorrentes 
de alterações genéticas são menos frequentes. Outros adotam 20 ou 22 semanas. Quanto ao limite superior de idade gestacional, a maioria dos autores adota 34 semanas, período a partir do qual diminui consideravelmente a morbidade e a mortalidade neonatal e não se justificam as complicações maternas e fetais decorrentes da tocólise. Além disso, a administração do corticoide antenatal só é indicada até 34 semanas ${ }^{5,6}$.

\section{Hidratação}

Estudos randomizados demonstraram que a hidratação não reduz a taxa de partos prematuros ${ }^{7,8}$. No entanto, nos casos em que as contrações uterinas e alterações cervicais são pouco pronunciadas e há dúvida quanto ao diagnóstico do TPP, pode-se infundir por via IV soro fisiológico e glicosado a $5 \%$, em partes iguais, num total de $1.000 \mathrm{~mL}$. Se após uma hora persistirem as contrações uterinas, a terapêutica medicamentosa é introduzida.

\section{Uterolíticos}

As drogas uterolíticas são utilizadas para inibir o TPP há quase 50 anos. Durante todos esses anos, apenas duas drogas foram desenvolvidas especialmente para esse fim: a ritodrina (agonista beta-adrenérgico) e o atosiban (antagonista do receptor de ocitocina). As outras drogas foram adaptadas para a tocólise. Atualmente, há três tipos de uterolíticos mais utilizados: agonistas beta-adrenérgicos, bloqueadores do canal de cálcio e antagonistas do receptor de ocitocina. Outras drogas também utilizadas para inibir o TPP (sulfato de magnésio, inibidores de prostaglandinas e doadores de óxido nítrico) carecem de comprovação quanto à sua eficácia ou são responsáveis por efeitos colaterais importantes.

Para a escolha do uterolítico, devem ser levadas em conta a eficácia e a segurança. Quanto à eficácia, se o ideal é a diminuição da taxa de partos prematuros, a morbidade e a mortalidade neonatal, e não apenas o adiamento do parto em 48 a 72 horas ou 7 dias, como a maioria dos estudos relatam. Além disso, a droga deve ser segura para a mãe, feto e recém-nascido. Diante da incapacidade de inibir as contrações uterinas com determinado uterolítico, ele pode ser substituído por outro. No entanto, antes da utilização de um segundo tocolítico, deve-se excluir a presença de corioamnionite e de alterações da vitalidade fetal. Não existem estudos controlados sobre o uso repetido de uterolíticos para o tratamento do parto prematuro recorrente ${ }^{9}$, embora recomendemos que, quando necessária, a repetição da tocólise seja feita uma só vez.

\section{Agonistas beta-adrenérgicos}

Em 1961, pela primeira vez, foi proposto o emprego de um agente betamimético, a isoxsuprina, para inibir as
Quadro 2 - Condições para 0 uso de uterolíticos.

Período de latência do trabalho de parto (dilatação cervical $<3 \mathrm{~cm}$ )

Esvaecimento não pronunciado

Idade gestacional entre 22 e 34 semanas

Ausência de contraindicacõoes

Quadro 3 - Contraindicações para a tocólise.

\begin{tabular}{|l|}
\hline Morte fetal \\
Sofrimento fetal \\
Malformaç̃oes fetais incompatíveis com a vida \\
Restriç̃a do crescimento fetal \\
Rotura da bolsa das águas \\
Infecção amniótica \\
Descolamento prematuro de placenta \\
Placenta prévia sangrante \\
Síndromes hipertensivas \\
Diabetes insulino-dependente instável \\
Cardiopatias \\
Hipertireoidismo não compensado \\
Anemia falciforme \\
\hline
\end{tabular}

contrações uterinas ${ }^{10}$. Com ação predominantemente em receptores beta-1 (coração e intestinos), causa mais efeitos colaterais cardiovasculares e, portanto, deve ser evitada. Em seu lugar, a preferência deve ser dada às drogas de efeito predominantemente beta-2 (com ação em miométrio, vasos sanguíneos e bronquíolos), tais como a terbutalina, o salbutamol e a ritodrina. Dessas três, apenas a ritodrina está aprovada pela Food and Drug Administration (FDA) dos EUA para inibir o TPP. Ao atuarem em receptores da célula miometrial, determinam o seu relaxamento por diminuição do cálcio livre no interior das células. $\mathrm{O}$ mecanismo envolvido nesse efeito consiste na ativação da enzima adenil-ciclase, que catalisa a conversão do ATP em AMP cíclico. Este último, por sua vez, diminui o cálcio livre intracelular.

Em revisão da Cochrane, na qual foram incluídas 1.332 gestantes pertencentes a 11 estudos randomizados e placebo-controlados, verificou-se que os agonistas betaadrenérgicos evitam o parto prematuro nas primeiras 48 horas $(\mathrm{RR}=0,6$; IC95\% $=0,5-0,7)$ e possivelmente antes de 7 dias $(R R=0,6 \text {; IC95\%=0,4-1,0 })^{11}$. Foi observada, também, uma tendência, embora não significativa, a reduzir a síndrome do desconforto respiratório (SDR) dos recém-nascidos $(\mathrm{RR}=0,8 ; \mathrm{IC95 \%}=0,7-1,0)$ e nenhum efeito sobre a mortalidade neonatal $(\mathrm{RR}=1,0$; IC95\% $=0,4-$ $2,0)$. Entre as críticas deste estudo, destaca-se o fato de que vários estudos que participaram dessa metanálise não utilizaram o corticoide antenatal de rotina, o que pode ter influenciado nos resultados.

Os agonistas beta-adrenérgicos também agem em outros órgãos e, no sistema cardiovascular, contribuem para o aparecimento de dor torácica, taquicardia, dispneia, mal-estar e edema agudo de pulmões. Além disso, atuam no sistema nervoso central (SNC) e causam cefaleia, 
tonturas e tremores ${ }^{12}$. Tais efeitos colaterais contribuem para a descontinuidade do tratamento. Além disso, essas substâncias atravessam a placenta, tendo sido descritos inúmeros efeitos colaterais no feto e no recém-nascido, como taquicardia, hiperinsulinismo, hipoglicemia, hipocalemia e hipotensão arterial ${ }^{13}$.

Em virtude dos efeitos colaterais, alguns cuidados devem ser tomados por ocasião do uso de tais drogas: realizar eletrocardiograma materno prévio; controlar com cuidado o pulso e a pressão arterial, mantendo o pulso materno abaixo de $120 \mathrm{bpm}$; auscultar periodicamente os pulmões e coração; e monitorar os batimentos cardíacos fetais. É importante salientar que os efeitos colaterais cardiovasculares, como o edema agudo de pulmões, são mais frequentes em situações de hipervolemia materna, como no polidrâmnio, na gestação gemelar e em pacientes submetidas à infusão de grande quantidade de líquidos. É importante destacar que, diante da tocólise com beta-agonistas, a administração de líquidos não deve ultrapassar 2 L em 24 horas.

\section{Esquema terapêutico}

São diluídas cinco ampolas de terbutalina ( $1 \mathrm{amp}=0,5$ $\mathrm{mg}$ ) em soro glicosado a $5 \%(500 \mathrm{~mL})$, que são infundidas por via intravenosa, iniciando-se com $2,5 \mu \mathrm{g} / \mathrm{min}$ (10 gotas/min); a seguir, aumenta-se para 10 gotas/min a cada 20 minutos até um máximo de 80 gotas/min; uma vez obtida a dose mínima capaz de cessar as contrações, mantém-se o gotejamento por 24 horas.

Se as contrações não diminuírem em 6 horas ou a tocólise for necessária por mais de 24 horas, deve-se pesquisar a presença de corioamnionite ou insuficiência placentária. Após as 24 horas de administração da droga, diminuem-se 10 gotas a cada 20 minutos, até a sua suspensão total. A paciente deve ser mantida em repouso absoluto e sob vigilância por mais 24 horas e, caso as contrações retornem, utiliza-se o esquema intravenoso mais uma vez.

\section{Bloqueadores do canal de cálcio}

\section{Mecanismo de ação}

Inibem a entrada do cálcio extracelular através da membrana citoplasmática, impedem a liberação do cálcio intracelular do retículo sarcoplasmático e aumentam a saída do cálcio da célula miometrial. Quanto à eficácia, até o momento não existem estudos placebo-controlados que avaliem a eficácia dos bloqueadores de canal do cálcio para inibir o TPP; há apenas estudos comparativos com outras drogas, os quais devem ser interpretados com cautela.

Em metanálise de 9 estudos (679 gestantes), em que se comparou a nifedipina à terbutalina ou ritodrina, verificou-se que a nifedipina foi mais eficaz para inibir o TPP nas primeiras 48 horas $(\mathrm{OR}=1,5 \text {; IC95\% }=1,0-2,2)^{14}$. Posteriormente, o mesmo grupo de autores realizou nova metanálise com 12 estudos (1.029 gestantes), na qual se comparou o uso de bloqueadores do canal de cálcio a outras drogas uterolíticas (principalmente agonistas beta-adrenérgicos). Observou-se que os bloqueadores do canal de cálcio não reduziram significativamente o risco de parto prematuro nas primeiras 48 horas $(R R=0,8$; IC95\% $=0,6-1,0)$, mas conseguiram reduzir o risco quando considerado o intervalo de 7 dias para a ocorrência do parto $(\mathrm{RR}=0,7 ; \mathrm{IC} 95 \%=0,6-0,9)^{15}$. Segundo os resultados desse estudo, houve redução significativa da SDR, enterocolite necrosante e hemorragia cerebral nos recém-nascidos com a utilização dos bloqueadores do canal do cálcio. Dentre as críticas, destaca-se o fato de que dois estudos participantes da metanálise exerceram forte influência sobre os resultados e pertenciam ao mesmo grupo de autores. Além disso, não houve seguimento dos recém-nascidos após a alta do berçário.

Recentemente, foi realizada revisão sistemática do uso da nifedipina para inibir o TPP. Foram identificados 45 estudos sobre o tema. Entretanto, nessa revisão foram incluídos apenas 31, dos quais $77 \%$ apresentavam vieses de amostragem, de aferição, de seleção e confusão. Além disso, o próprio fabricante não indica o seu uso para a inibição do TPP ${ }^{16}$. Portanto, ainda há a necessidade de estudos clínicos controlados para que se determine com maior precisão a aplicabilidade dessas drogas.

Por serem vasodilatadores periféricos, os bloqueadores do canal de cálcio podem causar rubor facial, cefaleia, tonturas, palpitações e hipotensão arterial, sendo de menores intensidades do que com os agonistas beta-adrenérgicos. Nas doses habitualmente utilizadas para inibir o TPP, não há dados sobre os efeitos colaterais fetais.

A posologia ideal é desconhecida. Há vários esquemas que podem ser utilizados, entre eles: $30 \mathrm{mg} \mathrm{VO}$, seguida de $20 \mathrm{mg}$ VO a cada 4 ou 6 horas; $20 \mathrm{mg} \mathrm{VO}$, seguida de $20 \mathrm{mg}$ VO em 90 minutos e $10 \mathrm{mg} \mathrm{VO}$ a cada 20 minutos, num total de 4 doses.

\section{Antagonistas de receptor da ocitocina}

Atuam competindo com a ocitocina no seu receptor da célula miometrial e reduzem os efeitos fisiológicos desse hormônio ${ }^{17}$. Dentre tais drogas, destaca-se o atosibano, comumente utilizado na Europa. Estudos comparativos de tamanhos amostrais relevantes têm demonstrado que o atosiban é tão eficaz quanto a ritodrina, o salbutamol e a terbutalina para inibir o TPP, sendo, mais seguro e mais bem tolerado do que os agonistas beta-adrenérgicos ${ }^{18,19}$. Da mesma forma, a morbidade e mortalidade neonatal foram semelhantes nos grupos avaliados. 
Quanto à comparação do atosibano com o placebo, em revisão da Cochrane, na qual foram avaliados seis estudos com 1.695 gestantes, verificou-se que o primeiro elevou o risco de parto prematuro nas primeiras 48 horas após o início do tratamento $(\mathrm{RR}=2,5$; IC95\%=0,5-12,3), elevou o risco de nascimento antes da $28^{\mathrm{a}}$ semana $(\mathrm{R} R=2,2$; IC95\% $=0,8-6,3)$ e também antes da $37^{\mathrm{a}}$ semana $(\mathrm{RR}=1,1$; IC95\% $=0,9-1,3)$. No entanto, nenhum desses aumentos foi significativo ${ }^{20}$. A morbidade e a mortalidade neonatal foram semelhantes nos dois grupos. Há de se destacar que o emprego do corticoide antenatal variou amplamente entre os estudos. Além disso, dois estudos que fizeram parte dessa metanálise, com total 613 gestantes, revelaram que o atosibano foi associado a uma menor média de peso ao nascimento e a complicações neonatais ${ }^{21,22}$. Posteriormente, verificou-se que a randomização dos grupos foi inadequada, pois havia duas vezes mais mulheres com idade gestacional inferior a 26 semanas no grupo atosibano do que no grupo placebo, ou seja, os grupos não eram homogêneos. Nas mulheres com idade gestacional superior a 28 semanas, os resultados maternos e neonatais foram melhores no grupo atosibano.

Diante de tais controvérsias, a mesma revisão Cochrane demonstrou que o atosibano foi igualmente eficaz em comparação a agonistas beta-adrenérgicos, pois evitam o nascimento prematuro dentro das primeiras 48 horas $(\mathrm{RR}=0,9 ; \mathrm{IC} 95 \%=0,6-1,4)$ ou dentro dos primeiros 7 dias de tratamento $(\mathrm{RR}=0,9$; IC95\%=0,6-1,2). $\mathrm{O}$ atosibano também foi associado a porcentagens de efeitos colaterais maternos $(\mathrm{RR}=0,04$; IC95\% $=0,02-0,1)$ quando comparado aos agonistas beta-adrenérgicos.

Dentre os efeitos colaterais maternos mais observados, destacam-se: náuseas, tonturas e cefaleia. Não foram observados efeitos cardiovasculares. Em relação aos efeitos colaterais fetais e neonatais, há um único estudo no qual se demonstrou maior taxa de complicações neonatais com o uso de atosiban em prematuros extremos. Conforme já discutido anteriormente, a interpretação desses dados é discutível porque a randomização desses grupos foi inadequada ${ }^{22}$.

$\mathrm{O}$ atosibano pode ser administrado, inicialmente, em duas etapas: uma dose de ataque de $0,9 \mathrm{~mL}(6,75 \mathrm{mg})$ injetada diretamente na veia durante um minuto. Para a manutenção, infundem-se duas ampolas de $5 \mathrm{~mL}$ em 90 $\mathrm{mL}$ de SG5\% (solução de $100 \mathrm{~mL}$ ), IV, durante 3 horas na velocidade de $24 \mathrm{~mL} / \mathrm{h}(300 \mu \mathrm{g} / \mathrm{min})$; posteriormente, infundem-se os $28 \mathrm{~mL}$ restantes da solução anterior em $3 \mathrm{~h} 30 \mathrm{~min}$ na velocidade de $8 \mathrm{~mL} / \mathrm{h}$, totalizando $6 \mathrm{~h} 30 \mathrm{~min}$. Antes de continuar com a administração da droga, devem ser monitoradas as contrações uterinas. Em nossas observações, a administração da droga por 6 h30min é suficiente para inibir as contrações uterinas em $75 \%$ dos $\operatorname{casos}^{23}$.

Se as contrações persistem, é realizada a terceira etapa: solução IV de $90 \mathrm{~mL}$ de SG5\% com duas ampolas de $5 \mathrm{~mL}$ de atosiban na velocidade de $8 \mathrm{~mL} / \mathrm{h}$ até que totalize 48 horas desde o início do tratamento.

\section{Sulfato de magnésio}

Ainda não se tem conhecimento exato de como age o magnésio sobre as contrações uterinas, mas provavelmente compete com o cálcio, impedindo a sua entrada pela membrana da célula miometrial.

$\mathrm{Na}$ última revisão da Cochrane, a qual envolveu 190 gestantes, em comapração ao placebo, o sulfato de magnésio não reduziu o nascimento prematuro antes de 48 horas $(\mathrm{RR}=0,5$; IC95\% $=0,2-1,1)$ e nem a SDR $(\mathrm{RR}=1,0$; IC95\% $=0,9-1,2)^{24}$. No mesmo estudo, foi verificado que o sulfato de magnésio não foi melhor e nem pior do que outros uterolíticos (beta-adrenérgicos, bloqueadores de canais de cálcio e inibidores da ciclooxigenase). No entanto, o tamanho amostral foi pequeno e o corticioide antenatal também não foi utilizado em todos os casos.

Apesar dos riscos potenciais, poucos efeitos colaterais maternos são observados quando a concentração sérica de magnésio é mantida em níveis terapêuticos $(4 \mathrm{a} 6 \mathrm{mEq} / \mathrm{L}$. No entanto, na hipermagnesemia surgem náuseas, vômitos, cefaleia, distúrbios visuais, letargia, fraqueza muscular, diminuição de reflexos, hipotensão arterial, palpitações e depressão respiratória ${ }^{25}$. O magnésio atravessa facilmente a barreira placentária e a hipermagnesemia pode causar hiporreatividade e hipotonia fetal. Dessa maneira, a gestante deve ser cuidadosamente monitorada em relação à diurese, frequência respiratória e reflexos patelares. Além disso, deve-se avaliar a magnesemia materna a cada seis horas.

A dose preconizada é de $4 \mathrm{~g}$ diluídos em soro glicosado a $10 \%$ e infundidos IV em 20 minutos, como dose de ataque, seguidos de 2 a $3 \mathrm{~g} / \mathrm{h}$ até cessarem as contrações uterinas.

\section{Inibidores de prostaglandinas}

\section{Mecanismo de ação}

Atuam inibindo a ciclooxigenase, enzima responsável pela síntese de prostaglandinas a partir do ácido aracdônico. Dentre essas drogas, a indometacina é a mais utilizada para a inibição do TPP. Em revisão realizada pela Cochrane com apenas 70 casos, em comparação ao placebo, a indometacina reduziu o risco de parto em 48 horas $(R R=0,2 ; \mathrm{IC} 95 \%=0,03-1,2)$ e em 7 dias $(R R=0,4$; IC95\% $=0,1-1,6)$. Não houve diferença quanto aos resultados neonatais ${ }^{26}$. No mesmo estudo, a indometacina foi comparada a outros uterolíticos (agonistas beta-adrenérgicos e sulfato de magnésio) em 415 casos. A indometacina se revelou mais eficaz para evitar o parto em 48 horas 
( $\mathrm{RR}=0,5$; IC95\%=0,3-1,0), embora os resultados não tenham sido significativos. Portanto, em vista do tamanho pequeno da amostra em quando comparada ao placebo e dos resultados duvidosos quando comparada a outras drogas, a sua eficácia ainda é discutível.

Dentre os efeitos colaterais maternos, destacam-se: náuseas, vômitos e disfunção plaquetária. Dos efeitos colaterais fetais mais comumente relacionados, podem ser citados o oligoâmnio e o fechamento precoce do ducto arterioso, principalmente quando a droga é utilizada após a $32^{\mathrm{a}}$ semana $^{27,28}$. Outras complicações neonatais estão associadas à indometacina: enterocolite necrosante, leucomalácia e hemorragia intraventricular ${ }^{29}$.

O esquema mais comumente empregado é o de uma dose inicial de $100 \mathrm{mg}$ por via retal, seguido de $25 \mathrm{mg}$ por via oral a cada 6 horas, por um período máximo de 48 horas para idades gestacionais de até 32 semanas. A ultrassonografia fetal e a ecocardiografia fetal devem ser realizadas periodicamente caso o tratamento exceda 48 horas.

\section{Doadores de óxido nítrico}

O óxido nítrico atua na atividade do sistema enzimático da quinase de cadeia leve da miosina e promove o relaxamento da célula miometrial. A nitroglicerina transdérmica é a mais utilizada na inibição do TPP. Ainda não existem evidências suficientes para o uso dessas drogas no TPP. Em estudo randômico com 153 parturientes entre a $24^{\mathrm{a}}$ e a $32^{\mathrm{a}}$ semana, a nitroglicerina foi comparada ao placebo. Entre as mulheres que utilizaram a nitroglicerina $(\mathrm{n}=74)$, houve risco neonatal mais baixo (hemorragia intraventricular, leucomalácia, complicações pulmonares e enterocolite necrosante) quando comparado ao placebo $(\mathrm{n}=79)(\mathrm{RR}=0,29$; IC95\%=0,08-1,00). Não foi observada diferença significativa entre os dois grupos para a diminuição do risco de parto antes da $28^{a}$ semana e nem de prolongamento da gestação por sete dias ${ }^{30}$. Em outro estudo com 238 parturientes, comparou-se o óxido nítrico a agonistas beta-adrenérgicos (salbutamol ou ritodrina). A inibição do TPP foi mais eficaz com os agonistas beta-adrenérgicos ${ }^{31}$.

Os efeitos colaterais observados com o uso de óxido nítrico foram: a hipotensão arterial materna, tonturas e palpitações. Até o momento, não há relatos de efeitos colaterais fetais e neonatais.

Embora possa ser utilizada a via intravenosa, a mais utilizada é a via transdérmica por meio de adesivos de nitroglicerina. Apesar de a dose ideal ainda não ser conhecida, recomenda-se a via transdérmica por meio de adesivo de $10 \mathrm{mg}$ aplicado na pele do abdome. Após uma hora, caso persistam as contrações, aplica-se mais um adesivo, sendo que ambos podem permanecer no local durante 24 horas e depois removidos ${ }^{32}$.

\section{Antibióticos}

Estudos mais recentes demonstram que, durante o TPP, a utilização de antibióticos de largo espectro na ausência de infecção clínica ou bacteriúria assintomática não adia o parto.

Em estudo de metanálise limitado a gestações de até 34 semanas, demonstrou-se que o uso de antibióticos não prolonga a gestação ${ }^{33}$. Além disso, há suspeitas de que antibioticoterapia cause comprometimento fetal. No estudo ORACLE II, que comparou o uso de antibióticos com placebo em mulheres em TPP e membranas intactas, verificou-se que crianças submetidas ao uso de antibióticos intraútero apresentaram mais deficiências funcionais aos sete anos do que aquelas que receberam o placebo ${ }^{34}$.

Deve ser lembrado que, durante o TPP, é importante instituir a profilaxia para a infecção neonatal pelo estreptococo do Grupo B, a menos que se disponha de uma cultura vaginal e retal negativas com menos de cinco semanas. Para a profilaxia, é recomendado o seguinte esquema: penicilina $G$ cristalina -5 milhões de UI - IV de ataque e 2,5 milhões de UI - IV 4/4h até o nascimento, ou ampicilina 2,0 g IV de ataque e 1,0 g IV $4 / 4 \mathrm{~h}$ até o nascimento. São necessárias pelo menos duas doses de antibióticos com intervalo de quatro horas antes do nascimento.

Em caso de alergia à penicilina, e não havendo risco para anafilaxia, emprega-se cefazolina $2 \mathrm{~g}$ IV, seguida de $1 \mathrm{~g}$ IV de 8/8h até o parto. Se houver risco para anafilaxia: opta-se por clindamicina $900 \mathrm{mg}$ IV de 8/8h ou eritromicina $500 \mathrm{mg}$ IV de 6/6h até o parto. Se houver resistência à clindamicina ou eritromicina, ou diante de suscetibilidade desconhecida: vancomicina $1 \mathrm{~g}$ IV de 12/12h até o parto.

\section{Corticoterapia}

Recomendada entre a $26^{a}$ e a $34^{a}$ semana de gestação. É utilizada a betametasona sob a dose de $12 \mathrm{mg}$ por via intramuscular ao dia, com intervalo de 24 horas num total de duas aplicações. O efeito máximo inicia-se após 24 horas e persiste por 7 dias. Geralmente, é utilizado apenas um único ciclo de corticoide e, excepcionalmente, dois, em virtude dos efeitos colaterais para o feto.

\section{Manutenção pós-tocólise}

Ainda não existem estudos com tamanhos amostrais adequados e bem conduzidos sobre a utilização de uterolíticos após o sucesso da tocólise. Em revisão da Cochrane na qual foram avaliados 11 estudos randomizados com agonistas beta-adrenérgicos e placebo, não se observaram diferenças significativas entre os grupos quanto ao 
prolongamento da gestação ${ }^{35}$. Outros estudos em que foram avaliados o sulfato de magnésio, a nifedipina e o antagonista da ocitocina também não demonstraram vantagens em relação ao placebo ${ }^{36-38}$.

É possível que a progesterona possa trazer algum benefício para a manutenção da gestação após a tocólise. Em estudo em avaliou o emprego do caproato de 17-alpha-hidroxiprogesterona (341 mg, IM 2 vezes por semana), na pós-tocólise com atosibano, entre a $25^{\mathrm{a}}$ a $33^{\mathrm{a}}$ semana, observou-se que o encurtamento do colo avaliado pela ultrassonografia foi maior no grupo sem o medicamento $(n=30)$ após o $7^{\circ}$ e $21^{\circ}$ dias quando comparado ao grupo progesterona $(\mathrm{n}=30)$. No grupo progesterona também houve menor risco de recidiva do TPP $(R R=0,1$; IC95\% $=0,04-0,5)^{39}$.

Em outro estudo, foi comparada a utilização de 400 $\mathrm{mg} / \mathrm{d}$ de progesterona natural pela via vaginal $(\mathrm{n}=37)$ e nenhum tratamento $(n=33)$ após tocólise com sulfato de magnésio. Embora a progesterona tenha prolongado de maneira significativa o período de latência de novo episódio de trabalho de parto (média de 12 dias), não foi observada diferença significativa entre os dois grupos quanto à recorrência do TPP ${ }^{40}$.

\section{Conclusões}

Os uterolíticos disponíveis na atualidade permitem abolir as contrações uterinas por algum tempo, o suficiente para a realização da corticoterapia antenatal e/ou para o transporte seguro da gestante para outro serviço.

A escolha do uterolítico deve ser baseada principalmente em sua eficácia e segurança. Quanto à eficácia, destacam-se os agonistas beta-adrenérgicos, os bloqueadores do canal de cálcio e os antagonistas de receptor da ocitocina. Em relação à segurança, os antagonistas do receptor da ocitocina apresentam vantagens em relação aos outros. No entanto, o seu custo para o tratamento é elevado. As outras drogas (sulfato de magnésio, inibidores de prostaglandinas e doadores de óxido nítrico) apresentam eficácias e seguranças discutíveis e, portanto, devem ser evitadas.

$\mathrm{Na}$ ausência de sinais clínicos de infecção, não é recomendado o uso de antibióticos de largo espectro com o intuito de prolongar a gestação. Quando indicada, deve ser realizada apenas a antibioticoterapia profilática para a infecção neonatal pelo estreptococo do Grupo B.

\section{Referências}

1. McPheeters ML, Miller WC, Hartmann KE, Savitz DA, Kaufman JS, Garrett JM, et al. The epidemiology of threatened preterm labor: a prospective cohort study. Am J Obstet Gynecol. 2005; 192(4): 1325-9

2. ACOG Committee on Practice Bulletins. American College of Obstetricians and Gynecologist. Clinical management guidelines for obstetrician-gynecologist. Number 43, May 2003. Management of preterm labor. Obstet Gynecol. 2003;101(5 Pt 1):1039-47.

3. Gomez R, Romero R, Medina L, Nien JK, Chaiworapongsa T, Carstens $M$, et al. Cervicovaginal fibronectin improves the prediction of preterm delivery based on sonographic cervical length in patients with preterm uterine contractions and intact membranes. Am J Obstet Gynecol. 2005;192(2):350-9.

4. Bittar RE, Pereira PP, Liao AW. Prematuridade. In: Zugaib M, editor. Obstetrícia. Barueri: Manole; 2008. p. 645-66.

5. Goldenberg RL. The management of preterm labor. Obstet Gynecol. 2002; 100(5 Pt 1):1020-37.

6. Korenbrot CC, Aalto LH, Laros RK Jr. The cost effectiveness of stopping preterm labor with beta-adrenergic treatment. N Engl J Med. 1984;310(1 1):691-6.

7. Pircon RA, Strassner HT, Kirz DS, Towers CV. Controlled trial of hydration and bed rest versus bed rest alone in the evaluation of preterm uterine contractions. Am J Obstet Gynecol. 1989;161(3):775-9.

8. Guinn DA, Goepfert AR, Owen J, Brumfield C, Hauth JC. Management options in women with preterm uterine contractions: a randomized clinical trial. Am J Obstet Gynecol. 1997;177(4):814-8.

9. Simhan $\mathrm{H}$, Caritis $\mathrm{S}$. Inhibition of acute preterm labor [serial on the Internet]. Up to Date, 2009 [cited 2009 Jan 10]. Available from: http://www.uptodate.com/patients/content/topic. do?topicKey $=\sim x h T x x k O 9 Y 6 X b . O v$.

10. Bishop EH, Woutersz TB. Isoxsuprine, a myometrial relaxant: a preliminary report. Obstet Gynecol. 1961;17(4): 442-6.

11. Anotayanonth S, Subhedar NV, Garner P, Neilson JP, Harigopal $\mathrm{S}$. Betamimetics for inhibiting preterm labour. Cochrane Database Syst Rev. 2004;(4):CD004352.

12. Gyetvai K, Hannah ME, Hodnett ED, Ohlsson A. Tocolytics for preterm labor: a systematic review. Obstet Gynecol. 1999;94(5 $\mathrm{Pt}$ 2):869-77.

13. Golichowski AM, Hathaway DR, Fineberg N, Peleg D. Tocolytic and hemodynamic effects of nifedipine in the ewe. Am J Obstet Gynecol. 1985;151(8):1134-40.

14. Tsatsaris V, Papatsonis D, Goffinet F, Dekker G, Carbonne B. Tocolysis with nifedipine or beta-adrenergic agonists: a metaanalysis. Obstet Gynecol. $2001 ; 97(5$ Pt 2):840.

15. King JF, Flenady VJ, Papatsonis DN, Dekker GA, Carbonne B. Calcium channel blockers for inhibiting preterm labour. Cochrane Database Syst Rev. 2003;(1)CD002255.

16. Lamont RF, Khan KS, Beattie B, Cabero Roura L, Di Renzo GC, Dudenhausen JW, et al. The quality of nifedipine studies used to assess tocolytic efficacy: a systematic review. J Perinat Med. 2005;33(4):287-95.

17. Phaneuf S, Asboth G, MacKenzie IZ, Melin P, López Bernal A. Effect of oxytocin antagonists on the activation of human myometrium in vitro: atosiban prevents oxytocin-induced desensitization. Am J Obstet Gynecol. 1994;171(6): 1627-34. 
18. Moutquin JM, Sherman D, Cohen H, Mohide PT, Hochner-Celnikier $D$, Fejgin $M$, et al. Double-blind, randomized, controlled trial of atosiban and ritodrine in the treatment of preterm labor: a multicenter effectiveness and safety study. Am J Obstet Gynecol. 2000; 182(5): $1191-9$.

19. The Worldwide Atosiban versus Beta-agonists Study Group. Effectiveness and safety of the oxytocin antagonist atosiban versus beta-adrenergic agonists in the treatment of preterm labour. BJOG. 2001;108(2):133-42.

20. Papatsonis D, Flenady V, Cole S, Liley H. Oxyłocin receptor antagonists for inhibiting preterm labour. Cochrane Database Syst Rev. 2005;(3):CD004452.

21. Goodwin TM, Paul R, Silver H, Spellacy W, Parsons $M$, Chez $R$, et al. The effect of the oxytocin antagonist atosiban on preterm uterine activity in the human. Am J Obstet Gynecol. $1994 ; 170(2): 474-8$.

22. Romero R, Sibai BM, Sanchez-Ramos L, Valenzuela GJ, Veille JC, Tabor B, et al. An oxytocin receptor antagonist (atosiban) in the treatment of preterm labor: a randomized, double-blind, placebo-controlled trial with tocolytic rescue. Am J Obstet Gynecol. 2000; 182(5): 1173-83.

23. Cabar FR, Bittar RE, Gomes CM, Zugaib M. O atosibano como agente tocolítico: uma nova proposta de esquema terapêutico. Rev Bras Ginecol Obstet. 2008;30(2):87-92.

24. Crowther CA, Hiller JE, Doyle LW. Magnesium sulphate for preventing preterm birth in threatened preterm labour. Cochrane Database Syst Rev. 2002;(4):CD001060.

25. Macones GA, Sehdev HM, Berlin M, Morgan MA, Berlin JA. Evidence for magnesium sulfate as a tocolytic agent. Obstet Gynecol Surv. 1997;52(10):652-8.

26. King J, Flenady V, Cole S, Thornton S. Cyclo-oxygenase (COX) inhibitors for treating preterm labour. Cochrane Database Syst Rev. 2005;(2):CD001992.

27. Sawdy R, Lye S, Fisk NM, Bennett PR. A double-blind randomized study of fetal side effects during and after the short-term maternal administration of indomethacin, sulindac, and nimesulide for the treatment of preterm labor. Am J Obstet Gynecol. 2003;188(4): 1046-51

28. Holmes RP, Stone PR. Sever oligohydramnios induced by cyclooxygenase-2 inhibitor nimesulide. Obstet Gynecol. 2000;96/5 Pt 2):810-1.

29. Doyle NM, Gardner MO, Wells L, Qualls C, Papile LA. Outcome of very low birth weight infants exposed to antenatal indomethacin for tocolysis. J Perinatol. 2005;25(5): 336-40.

30. Smith GN, Walker MC, Ohlsson A, O'Brien K, Windrim R; Canadian Preterm Labour Nitroglycerin Trial Group. Randomized double-blind placebo-controlled trial of transdermal nitroglycerin for preterm labor. Am J Obstet Gynecol. 2007;196(1): 37.el-8.

31. Bisits A, Madsen G, Knox M, Gill A, Smith R, Yeo G. The Randomized Nitric Oxide Tocolysis Trial (RNOTT) for the treatment of preterm labor. Am J Obstet Gynecol. 2004;191 (3):683-90.

32. Smith GN, Walker MC, McGrath M. Randomised, double-blind, placebo controlled pilot study assessing nitroglycerin as a tocolytic. Br J Obstet Gynecol. 1999; 106(7):736-9.

33. Hutzal CE, Boyle EM, Kenyon SL, Nash JV, Winsor S, Taylor DJ, et al. Use of antibiotics for the treatment of preterm parturition and prevention of neonatal morbidity: a metaanalysis. Am J Obstet Gynecol. 2008;199(6):620.e1-8.

34. Kenyon S, Pike K, Jones DR, Brocklehurst P, Marlow N, Salt $A$, et al. Childhood outcomes after prescription of antibiotics to pregnant women with spontaneous preterm labour: 7-year follow-up of the ORACLE II trial. Lancet. 2008;372(9646): 1319-27.

35. Dodd JM, Crowther CA, Dare MR, Middleton P. Oral betamimetics for maintenance therapy after threatened preterm labour. Cochrane Database Syst Rev. 2006;(1):CD003927.

36. Lyell DJ, Pullen KM, Mannan J, Chitkara U, Druzin ML, Caughey $A B$, et al. Maintenance nifedipine tocolysis compared with placebo: a randomized controlled trial. Obstet Gynecol. $2008 ; 112(6): 1221-6$

37. Crowther CA, Moore V. Magnesium for preventing birth after threatened preterm labour. Cochrane Database Syst Rev. 2000;(2):CD000940.

38. Valenzuela GJ, Sanchez-Ramos L, Romero R, Silver HM, Koltun WD, Millar $L$, et al. Maintenance treatment of preterm labor with oxytocin antagonist atosiban. The Atosiban PTL-098 Study Group. Am J Obstet Gynecol. 2000; 182(5): 1 184-90.

39. Facchinetti F, Paganelli S, Comitini G, Dante G, Volpe A. Cervical length changes during preterm cervical ripening: effects of 17 alpha-hydroxyprogesterone caproate. Am J Obstet Gynecol. 2007; 196(5):453.e 1-4

40. Borna S, Sahabi N. Progesterone for maintenance tocolytic therapy after threatened preterm labour: a randomised controlled trial. Aust N Z J Obstet Gynaecol. 2008;48(1):58-63. 